\title{
Fixed point theorems for expanding mappings in partial metric spaces
}

\author{
Xianjiu Huang, Chuanxi Zhu, Xi Wen
}

\begin{abstract}
In this paper, we define expanding mappings in the setting of partial metric spaces analogous to expanding mappings in metric spaces. We also obtain some results for two mappings to the setting of partial metric spaces.
\end{abstract}

\section{Introduction and Preliminaries}

In 1994, Matthews [10] introduced the notion of partial metric space as a part of the study of denotational semantics of dataflow networks. He generalized the concept of metric space in the sense that the distance from a point to itself need not be equal to zero. Such metrics are useful in modeling partially defined information, which often appears in Computer Science. In the same reference, the contraction fixed point theorem was extended to partial metric spaces. This highlights an additional feature: the fixed point has self-distance 0 . Although trivial in metric spaces, this can be useful for reasoning about posets appearing in Computer Science. For, when a computable function is shown to be a contraction, the partial metric extension of the contraction fixed point theorem can be used to prove that the unique fixed point, which is the program output, will be totally computed; see [10] for details. Further applications of partial metrics to problems in theoretical Computer Science were discussed in [2-3, 14-17].

In 1984, Wang et.al [19] introduced the concept of expanding mappings and proved some fixed point theorems in complete metric spaces. In 1992,

Key Words: expanding mappings; fixed point theorem; partial metric spaces

2010 Mathematics Subject Classification: 47H10

Received: November, 2010.

Revised: November, 2011.

Accepted: February, 2012. 
Daffer and Kaneko[4] defined an expanding condition for a pair of mappings and proved some common fixed point theorems for two mappings in complete metric spaces.

In this paper, we define expanding mappings in the setting of partial metric spaces analogous to expanding mappings in complete metric spaces(see Wang et.al [19]). We also extend a result of Daffer and Kaneko[4] for two mappings to the setting of partial metric spaces.

Throughout this paper the letters $R, R^{+}, N$ will denote the set of real numbers, nonnegative real numbers and natural numbers, respectively. We use the following definitions in the proof of our main theorems.

We recall that given a (nonempty) set $X$, a function $p: X \times X \rightarrow R^{+}$is called a partial metric if and only if for all $x, y, z \in X$ :

$\left(p_{1}\right) x=y \Leftrightarrow p(x, x)=p(x, y)=p(y, y)$;

$\left(p_{2}\right) p(x, x) \leq p(x, y)$;

$\left(p_{3}\right) p(x, y)=p(y, x)$;

$\left(p_{4}\right) p(x, z) \leq p(x, y)+p(y, z)-p(y, y)$.

A partial metric space is a pair $(X, p)$ such that $X$ is a nonempty set and $p$ is a partial metric on $X$. It is clear that, if $p(x, y)=0$, then from $\left(p_{1}\right)$ and $\left(p_{2}\right)$ $x=y$. But if $x=y, p(x, y)$ may not be 0 . A basic example of a partial metric space is the pair $\left(R^{+}, p\right)$, where $p(x, y)=\max \{x, y\}$ for all $x, y \in R^{+}$. Other examples of partial metric spaces which are interesting from a computational point of view may be found in $[10-12,18]$.

There are some generalizations of partial metrics. For example, O'Neill[12] proposed one significant change to Matthews' definition of the partial metric, and that was extend their range from $R^{+}$to $R$. According to [12], the partial metrics in the O'Neill sense will be called dualistic partial metric and a pair $(X, p)$ such that $X$ is a nonempty set and $p$ is a dualistic partial metric on $X$ will be called a dualistic partial metric space. In this way, O'Neill developed several connections between partial metrics and the topological aspects of domain theory. Moreover, the pair $(R, p)$, where $p(x, y)=x \vee y$ for all $x, y \in R$, provides a paradigmatic example of a dualistic partial metric space that is not a partial metric space. Also, Heckmann [6] generalized it by omitting small self-distance axiom $p(x, x) \leq p(x, y)$. The partial metric of Heckmann sense is called weak partial metric. The inequality $2 p(x, y) \geq p(x, x)+p(y, y)$ is satisfied for all $x, y$ in a weak partial metric space.

Each partial metric $p$ on $X$ generates a $\mathcal{T}_{0}$ topology $\mathcal{T}(p)$ on $X$ which has as a base the family of open $p$-balls $\left\{B_{p}(x, \varepsilon): x \in X ; \varepsilon>0\right\}$, where $\left\{B_{p}(x, \varepsilon)=\{y \in X: p(x, y)<p(x, x)+\varepsilon\}\right.$ for all $x \in X$ and $\varepsilon>0$.

From this fact it immediately follows that a sequence $\left\{x_{n}\right\}$ in a partial metric space $(X, p)$ converges to a point $x \in X$ if and only if $p(x, x)=$ $\lim _{n \rightarrow \infty} p\left(x, x_{n}\right)$. Following [9] (compare [11]), a sequence $\left\{x_{n}\right\}$ in a partial metric 
space $(X, p)$ is called a Cauchy sequence if there exists $\lim _{n, m \rightarrow \infty} p\left(x_{n}, x_{m}\right)$. A partial metric space $(X, p)$ is said to be complete if every Cauchy sequence $\left\{x_{n}\right\}$ in $X$ converges, with respect to $\mathcal{T}(p)$, to a point $x \in X$ such that $p(x, x)=\lim _{n, m \rightarrow \infty} p\left(x_{n}, x_{m}\right)$. The continuity of the self-maps in the partial metric spaces is, in fact, the sequential continuity. If $f: X \rightarrow X$, where $(X, p)$ is a partial metric space, then $f$ is continuous at the point $a \in X$ if, for every sequence $x_{n} \in X$, which converges in the partial metric $p$ to $a$, the sequence $f x_{n}$ converges to $f a$, i.e.,

$$
p(a, a)=\lim _{n \rightarrow \infty} p\left(x_{n}, a\right) \Rightarrow p(f a, f a)=\lim _{n \rightarrow \infty} p\left(f x_{n}, f a\right) .
$$

It is easy to see that, every closed subset of a complete partial metric space is complete.

If $p$ is a partial metric on $X$, then the function $p^{s}: X \times X \rightarrow R^{+}$given by

$$
p^{s}(x, y)=2 p(x, y)-p(x, x)-p(y, y)
$$

is a metric on $X$.

Definition 1.1 Let $(X, d)$ be a partial metric space and $T: X \rightarrow X$. Then $T$ is called a expanding mapping, if for every $x, y \in X$ there exists a number $k>1$ such that $p(T x, T y) \geq k p(x, y)$.

Definition 1.2 Two self mappings $f$ and $g$ of a partial metric space $(X, d)$ are said to be commuting if $f g x=g f x$ for all $x \in X$.

Definition 1.3 Let $f$ and $g$ be self mappings of a set $X$ (i.e., $f, g: X \rightarrow$ $X)$. If $w=f x=g x$ for some $x$ in $X$, then $x$ is called a coincidence point of $f$ and $g$, and $w$ is called a point of coincidence of $f$ and $g$. Self mappings $f$ and $g$ are said to be weakly compatible if they commute at their coincidence point; i.e., if $f x=g x$ for some $x \in X$, then $f g x=g f x$.

Weakly compatible mappings are more general than that of commuting but neither implication is reversible.

The following lemma will be useful in what follows; see [10,11].

Lemma 1.1 Let $(X, p)$ be a partial metric space.

(1) $\left\{x_{n}\right\}$ is a Cauchy sequence in $(X, p)$ if and only if it is a Cauchy sequence in the metric space $\left(X, p^{s}\right)$.

(2) A partial metric space $(X, p)$ is complete if and only if the metric space $\left(X, p^{s}\right)$ is complete. Furthermore $\lim _{n \rightarrow \infty} p^{s}\left(a, x_{n}\right)=0$ if and only if $p(a, a)=$ $\lim _{n \rightarrow \infty} p\left(a, x_{n}\right)=\lim _{n, m \rightarrow \infty} p\left(x_{n}, x_{m}\right)$.

\section{Main results}

In this section, we shall establish some fixed point theorems concerning expanding maps. The following lemma will be useful. 
Lemma 2.1 Let $(X, p)$ be a partial metric space and $\left\{x_{n}\right\}$ be a sequence in $X$. If there exists a number $k \in(0,1)$ such that

$$
p\left(x_{n+1}, x_{n}\right) \leq k p\left(x_{n}, x_{n-1}\right), n=1,2, \cdots
$$

then $\left\{x_{n}\right\}$ is a Cauchy sequence in $X$.

Proof By the simple induction with the condition (2.1), we have

$$
p\left(x_{n+1}, x_{n}\right) \leq k p\left(x_{n}, x_{n-1}\right) \leq k^{2} p\left(x_{n-1}, x_{n-2}\right) \leq \cdots \leq k^{n} p\left(x_{1}, x_{0}\right) .
$$

On the other hand, since

$$
\max \left\{p\left(x_{n}, x_{n}\right), p\left(x_{n+1}, x_{n+1}\right)\right\} \leq p\left(x_{n}, x_{n+1}\right)
$$

then from (2.2) we have

$$
\max \left\{p\left(x_{n}, x_{n}\right), p\left(x_{n+1}, x_{n+1}\right)\right\} \leq k^{n} p\left(x_{1}, x_{0}\right) .
$$

Therefore,

$$
\begin{aligned}
p^{s}\left(x_{n}, x_{n+1}\right) & =2 p\left(x_{n}, x_{n+1}\right)-p\left(x_{n}, x_{n}\right)-p\left(x_{n+1}, x_{n+1}\right) \\
& \leq 2 p\left(x_{n}, x_{n+1}\right)+p\left(x_{n}, x_{n}\right)+p\left(x_{n+1}, x_{n+1}\right) \leq 4 k^{n} p\left(x_{1}, x_{0}\right) .
\end{aligned}
$$

This shows that $\lim _{n \rightarrow \infty} p^{s}\left(x_{n}, x_{n+1}\right)=0$. Now we have

$$
\begin{aligned}
p^{s}\left(x_{n}, x_{n+l}\right) & =p^{s}\left(x_{n}, x_{n+1}\right)+p^{s}\left(x_{n+1}, x_{n+2}\right)+\cdots+p^{s}\left(x_{n+l-1}, x_{n+l}\right) \\
& \leq 4 k^{n} p\left(x_{1}, x_{0}\right)+4 k^{n+1} p\left(x_{1}, x_{0}\right)+\cdots+4 k^{n+l-1} p\left(x_{1}, x_{0}\right) \\
& \leq \frac{4 k^{n}}{1-k} p\left(x_{1}, x_{0}\right) .
\end{aligned}
$$

This shows that $\left\{x_{n}\right\}$ is a Cauchy sequence in metric spaces $\left(X, p^{s}\right)$, then from Lemma 1.1, $\left\{x_{n}\right\}$ is a Cauchy sequence in partial metric spaces $(X, p)$.

Theorem 2.1 Let $(X, p)$ be a complete partial metric space and $T: X \rightarrow X$ be a surjection. Suppose that there exist $a_{1}, a_{2}, a_{3} \geq 0$ with $a_{1}+a_{2}+a_{3}>1$ such that

$$
p(T x, T y) \geq a_{1} p(x, y)+a_{2} p(x, T x)+a_{3} p(y, T y), \text { for all } x, y \in X, x \neq y .
$$

Then $T$ has a fixed point in $X$.

Proof Let $x_{0} \in X$. Since $T$ is surjective, choose $x_{1} \in X$ such that $T x_{1}=$ $x_{0}$. Inductively, we can define a sequence $\left\{x_{n}\right\} \in X$ such that $x_{n-1}=T x_{n}$, $n=1,2, \cdots$. 
Without loss of generality, we assume that $x_{n-1} \neq x_{n}$ for all $n=1,2, \cdots$ (otherwise, if there exists some $n_{0}$ such that $x_{n_{0}-1}=x_{n_{0}}$, then $x_{n_{0}}$ is a fixed point of $T$ ).

It follows that from condition (2.4)

$$
\begin{aligned}
p\left(x_{n-1}, x_{n}\right) & =p\left(T x_{n}, T x_{n+1}\right) \\
& \geq a_{1} p\left(x_{n}, x_{n+1}\right)+a_{2} p\left(x_{n}, T x_{n}\right)+a_{3} p\left(x_{n+1}, T x_{n+1}\right) \\
& =a_{1} p\left(x_{n}, x_{n+1}\right)+a_{2} p\left(x_{n}, x_{n-1}\right)+a_{3} p\left(x_{n+1}, x_{n}\right) \\
& \left(1-a_{2}\right) p\left(x_{n-1}, x_{n}\right) \geq\left(a_{1}+a_{3}\right) p\left(x_{n+1}, x_{n}\right)
\end{aligned}
$$

If $a_{1}+a_{3}=0$, then $a_{2}>1$. The above inequality implies that a negative number is greater than or equal to zero. That is impossible. So, $a_{1}+a_{3} \neq 0$ and $\left(1-a_{2}\right)>0$. Therefore,

$$
p\left(x_{n+1}, x_{n}\right) \leq h p\left(x_{n-1}, x_{n}\right)
$$

where $h=\frac{1-a_{2}}{a_{1}+a_{3}}<1$. By Lemma 2.1, $\left\{x_{n}\right\}$ is a Cauchy sequence in $X$. Since $(X, p)$ is complete, then from Lemma $1.1\left(X, p^{s}\right)$ is complete and so the sequence $\left\{x_{n}\right\}$ is converges in the metric space $\left(X, p^{s}\right)$, that is, there exists a point $z \in X$ such that

$$
\lim _{n \rightarrow \infty} p^{s}\left(x_{n}, z\right)=0 .
$$

Consequently, we can find $u \in X$ such that $z=T u$. Again from Lemma 1.1 , we have

$$
p(z, z)=\lim _{n \rightarrow \infty} p\left(x_{n}, z\right)=\lim _{n, m \rightarrow \infty} p\left(x_{n}, x_{m}\right) .
$$

Moreover, since $\left\{x_{n}\right\}$ is a Cauchy sequence in the metric space $\left(X, p^{s}\right)$, we have

$$
\lim _{n, m \rightarrow \infty} p^{s}\left(x_{n}, x_{m}\right)=0 .
$$

On the other hand, since

$$
\max \left\{p\left(x_{n}, x_{n}\right), p\left(x_{n+1}, x_{n+1}\right)\right\} \leq p\left(x_{n}, x_{n+1}\right)
$$

then by the simple induction with (2.5) we have

$$
\max \left\{p\left(x_{n}, x_{n}\right), p\left(x_{n+1}, x_{n+1}\right)\right\} \leq h^{n} p\left(x_{1}, x_{0}\right) .
$$

Hence, we have $\lim _{n \rightarrow \infty} p\left(x_{n}, x_{n}\right)=0$. Thus from the definition $p^{s}$, we have

$$
\lim _{n, m \rightarrow \infty} p\left(x_{n}, x_{m}\right)=0 .
$$

Therefore, from (2.6) we have

$$
p(z, z)=\lim _{n \rightarrow \infty} p\left(x_{n}, z\right)=\lim _{n, m \rightarrow \infty} p\left(x_{n}, x_{m}\right)=0 .
$$


Now, we show that $u=z$. From condition (2.4) we obtain

$$
p\left(x_{n}, z\right)=p\left(T x_{n+1}, T u\right) \geq a_{1} p\left(x_{n+1}, u\right)+a_{2} p\left(x_{n+1}, x_{n}\right)+a_{3} p(u, T u)
$$

which implies that as $n \rightarrow \infty$

$$
0=p(z, z) \geq\left(a_{1}+a_{3}\right) p(u, z) .
$$

Hence, $p(u, z)=0$, that is $u=z=T u$.

This gives that $z$ is a fixed point of $T$. This completes the proof.

Remark 2.1 Setting $a_{2}=a_{3}=0$ and $a_{1}=\lambda$ in Theorem 2.1, we can obtain the following result.

Corollary 2.1 Let $(X, p)$ be a complete partial metric space and $T: X \rightarrow$ $X$ be a surjection. Suppose that there exists a constant $\lambda>1$ such that

$$
p(T x, T y) \geq \lambda p(x, y), \text { for all } x, y \in X .
$$

Then $T$ has a unique fixed point in $X$.

Proof From Theorem 2.1, it follows that $T$ has a fixed point $z$ in $X$ by setting $a_{2}=a_{3}=0$ and $a_{1}=\lambda$ in condition (2.4).

Uniqueness. Suppose that $z \neq w$ is also another fixed point of $T$, then from condition (2.8), we obtain

$$
p(z, w)=p(T z, T w) \geq \lambda p(z, w)
$$

which implies $p(z, w)=0$, that is $z=w$. This completes the proof.

Corollary 2.2 Let $(X, p)$ be a complete partial metric space and $T: X \rightarrow$ $X$ be a surjection. Suppose that there exist a positive integer $n$ and a constant $\lambda>1$ such that

$$
p\left(T^{n} x, T^{n} y\right) \geq \lambda p(x, y), \text { for all } x, y \in X .
$$

Then $T$ has a unique fixed point in $X$.

Proof From Corollary 2.1, $T^{n}$ has a unique fixed point $z$. But $T^{n}(T z)=$ $T\left(T^{n} z\right)=T z$, so $T z$ is also a fixed point of $T^{n}$. Hence $T z=z, z$ is a fixed point of $T$. Since the fixed point of $T$ is also fixed point of $T^{n}$, the fixed point of $T$ is unique.

Theorem 2.2 Let $(X, p)$ be a complete partial metric space and $T: X \rightarrow$ $X$ be a continuous surjection. Suppose that there exist a constant $\lambda>1$ such that, for each $x, y \in X$,

$$
p(T x, T y) \geq \lambda u, \text { for some } u \in\{p(x, y), p(x, T x), p(y, T y)\}
$$


Then $T$ has a fixed point in $X$.

Proof Similar to the proof of Theorem 2.1, we can obtain a sequence $\left\{x_{n}\right\}$ such that $x_{n-1}=T x_{n}$.

Without loss of generality, we assume that $x_{n-1} \neq x_{n}$ for all $n=1,2, \cdots$ (otherwise, if there exists some $n_{0}$ such that $x_{n_{0}-1}=x_{n_{0}}$, then $x_{n_{0}}$ is a fixed point of $T$ ).

It follows that from condition (2.10)

$$
p\left(x_{n-1}, x_{n}\right)=p\left(T x_{n}, T x_{n+1}\right) \geq \lambda u_{n}
$$

where $u_{n}=\left\{p\left(x_{n}, x_{n+1}\right), p\left(x_{n}, x_{n-1}\right)\right\}$.

Now we have to consider the following two cases.

If $u_{n}=p\left(x_{n}, x_{n-1}\right)$, then

$$
p\left(x_{n-1}, x_{n}\right) \geq \lambda p\left(x_{n}, x_{n-1}\right)
$$

which implies $p\left(x_{n-1}, x_{n}\right)=0$, that is $x_{n-1}=x_{n}$. This is a contradiction.

If $u_{n}=p\left(x_{n}, x_{n+1}\right)$, then

$$
p\left(x_{n-1}, x_{n}\right) \geq \lambda p\left(x_{n}, x_{n+1}\right) .
$$

By Lemma 2.1, $\left\{x_{n}\right\}$ is a Cauchy sequence in $X$. Since $(X, p)$ is complete, the sequence $\left\{x_{n}\right\}$ converges to a point $z \in X$.

Since $T$ is continuous, it is clear that $z$ is a fixed point of $T$. This completes the proof.

Now, we give a common fixed point theorem of two weakly compatible mappings in partial metric spaces.

Theorem 2.3 Let $(X, p)$ be a partial metric space. Let $S$ and $T$ be weakly compatible self-mappings of $X$ and $T(X) \subseteq S(X)$. Suppose that there exists a constant $\lambda>1$ such that

$$
p(S x, S y) \geq \lambda p(T x, T y), \text { for all } x, y \in X .
$$

If one of the subspaces $T(X)$ or $S(X)$ is complete, then $S$ and $T$ have a unique common fixed point in $X$.

Proof Let $x_{0} \in X$. Since $T(X) \subseteq S(X)$, choose $x_{1}$ such that $y_{1}=S x_{1}=$ $T x_{0}$. In general, choose $x_{n+1}$ such that $y_{n+1}=S x_{n+1}=T x_{n}$. Then from (2.11),

$$
p\left(y_{n+1}, y_{n+2}\right)=p\left(T x_{n}, T x_{n+1}\right) \leq \frac{1}{\lambda} p\left(S x_{n}, S x_{n+1}\right)
$$




$$
=\frac{1}{\lambda} p\left(T x_{n-1}, T x_{n}\right)=\frac{1}{\lambda} p\left(y_{n}, y_{n+1}\right) .
$$

Thus, by Lemma 2.1, $\left\{y_{n}\right\}$ is a Cauchy sequence. Since $T(X) \subseteq S(X)$ and $T(X)$ or $S(X)$ is a complete subspace of $X$ then from Lemma $1.1\left(S(X), p^{s}\right)$ is complete and so the sequence $y_{n}=T\left(x_{n-1}\right) \subseteq S(X)$ is converges in the metric space $\left(S(X), p^{s}\right)$, that is, there exists a $z$ in $S(X)$ such that

$$
\lim _{n \rightarrow \infty} p^{s}\left(y_{n}, z\right)=0 \text {. }
$$

Consequently, we can find $u \in X$ such that $S u=z$. Again from Lemma 1.1 , we have

$$
p(S u, z)=p(z, z)=\lim _{n \rightarrow \infty} p\left(y_{n}, z\right)=\lim _{n, m \rightarrow \infty} p\left(y_{n}, y_{m}\right) .
$$

Moreover, since $\left\{y_{n}\right\}$ is a Cauchy sequence in the metric space $\left(S(X), p^{s}\right)$, we have

$$
\lim _{n, m \rightarrow \infty} p^{s}\left(y_{n}, y_{m}\right)=0 .
$$

On the other hand, since

$$
\max \left\{p\left(y_{n}, y_{n}\right), p\left(y_{n+1}, y_{n+1}\right)\right\} \leq p\left(y_{n}, y_{n+1}\right)
$$

then by the simple induction with (2.12) we have

$$
\max \left\{p\left(y_{n}, y_{n}\right), p\left(y_{n+1}, y_{n+1}\right)\right\} \leq\left(\frac{1}{\lambda}\right)^{n} p\left(y_{1}, y_{0}\right) .
$$

Hence, we have $\lim _{n \rightarrow \infty} p\left(y_{n}, y_{n}\right)=0$. Thus from the definition $p^{s}$, we have

$$
\lim _{n, m \rightarrow \infty} p\left(y_{n}, y_{m}\right)=0
$$

Therefore, from (2.13) we have

$$
p(S u, z)=p(z, z)=\lim _{n \rightarrow \infty} p\left(y_{n}, z\right)=\lim _{n, m \rightarrow \infty} p\left(y_{n}, y_{m}\right)=0 .
$$

Now, we show that $T u=z$. From condition (2.11)

$$
p\left(T u, T x_{n}\right) \leq \frac{1}{\lambda} p\left(S u, S x_{n}\right) .
$$

Proceeding to the limit as $n \rightarrow \infty$, we have $p(T u, z) \leq \frac{1}{\lambda} p(S u, z)=0$, which implies that $p(T u, z)=0$, that is $T u=z$. Therefore, $T u=S u=z$. Since $S$ and $T$ are weakly compatible, $S T u=T S u$, that is $S z=T z$. 
Now we show that $z$ is a common fixed point of $S$ and $T$. From condition

$$
p\left(S z, S x_{n}\right) \geq \lambda p\left(T z, T x_{n}\right) .
$$

Proceeding to the limit as $n \rightarrow \infty$, we have $p(S z, z) \geq \lambda p(T z, z)=$ $\lambda p(S z, z)$, which implies that $p(S z, z)=0$, that is $S z=z$. Hence $S z=T z=z$.

Uniqueness. Suppose that $z \neq w$ is also another common fixed point of $S$ and $T$, that is $S w=T w=w$. Then $p(z, w)=p(S z, S w) \geq \lambda p(T z, T w)=$ $\lambda p(z, w)$, this implies that $p(z, w)=0$, that is $z=w$. This completes the proof.

Now we give an example illustrating Theorem 2.3.

Example 2.1 Let $X=[0,1]$ and $p(x, y)=\max \{x, y\}$, then $(X, p)$ is a complete partial metric space. Let $S(x)=\frac{x}{2}, T(x)=\frac{x}{6}$ for all $x, y \in X$. Then $T(X) \subseteq S(X)$ and $S(X)$ is complete. Further, for all $x \in[0,1]$ with $x \geq y$ we have

$$
d(S x, S y)=\max \left\{\frac{x}{2}, \frac{y}{2}\right\}=\frac{x}{2} \geq \frac{\lambda}{6} d(T x, T y)
$$

for $1<\lambda<3$ and (2.11) is satisfied. Moreover, mappings $S$ and $T$ are weakly compatible at $x=0$ and 0 is the unique common fixed point. Thus all the conditions of Theorem 2.3 are satisfied.

Remark 2.2 In Theorem 2.3, the weak compatibility condition cannot be removed.

Indeed, letting $(X, p)$ be defined as in Example 2.1, define the mappings $S(x)=1-x, T(x)=\frac{1}{2}-\frac{x}{2}, x \in X$. Then $T(X) \subseteq S(X)$ and $S(X)$ is complete. Moreover, for all $x \in[0,1]$ with $x \geq y$ we have

$$
p(S x, S y)=\max \{1-x, 1-y\}=1-x \geq \lambda p(T x, T y)
$$

for $1<\lambda<2$ and (2.11) is satisfied. $S 1=T 1=0$ but $S T 1=1$ and $T S 1=\frac{1}{2}$, so $S$ and $T$ are not weakly compatible. It follows that except for the weakly compatibility of $S$ and $T$ all other hypotheses of Theorem 2.3 are satisfied. But they do not have a common fixed point. This shows that the weakly compatibility of $S$ and $T$ in Theorem 2.3 is an essential condition.

Daffer and Kaneko[4] prove a fixed point theorem for a pair of mappings. We extend their result in partial metric space, thus defining an expanding condition for a pair of mappings in Corollary 2.3 below.

Corollary 2.3 Let $(X, p)$ be a complete partial metric space. Let $S$ : $X \rightarrow X$ be a surjection and $T: X \rightarrow X$ be an injective. If $S$ and $T$ are commutative, and there exists a constant $\lambda>1$ such that

$$
p(S x, S y) \geq \lambda p(T x, T y), \text { for all } x, y \in X,
$$

then $S$ and $T$ have a unique common fixed point in $X$. 
Proof Note that mappings which commute are clearly weakly compatible and $S(X)$ is complete and $T(X) \subseteq S(X)$ in Corollary 2.3 since $S$ is surjective. Then, we can apply Theorem 2.3 that assures the existence of a unique common fixed point of $S$ and $T$ in $X$.

Acknowledgments The authors would like to express their sincere appreciation to the referees for their very helpful suggestions and many kind comments. This work is supported by the National Natural Science Foundation of China (11071108) and the Provincial Natural Science Foundation of Jiangxi, China (20114BAB201003) and supported partly by the Science and Technology Project of Educational Commission of Jiangxi Province, China (GJJ11294) and the Science Foundation of Nanchang University (Z04868, Z04877).

\section{References}

[1] I. Altun, F. Sola, H. Simsek, Generalized contractions on partial metric spaces, Topology Appl. 157(18) (2010), 2778-2785.

[2] M. Bukatin, J. Scott, Towards computing distances between programs via Scott domains, in: Logical Foundations of Computer Sicence, Lecture Notes in Computer Science (eds. S. Adian and A. Nerode), vol. 1234, Springer (Berlin, 1997), 33-43.

[3] M. Bukatin, S. Shorina, Partial metrics and co-continuous valuations, in: Foundations of Software Science and Computation Structures, Lecture Notes in Computer Science (ed. M. Nivat), vol. 1378, Springer (Berlin, 1998), 33-43.

[4] P. Z. Daffer, H. Kaneko, On expansive mappings, Math. Japonica. 37 (1992), 733-735.

[5] P. Fletcher, W. Lindgren, Quasi-Uniform Spaces, Marcel Dekker, New York, 1982.

[6] R. Heckmann, Approximation of metric spaces by partial metric spaces, Appl. Categ. Structures. 7 (1999), 71-83.

[7] G. Jungck, Common fixed points for noncontinuous nonself mappings on nonmetric spaces, Far East J. Math. Sci. 4 (2) (1996), 199-212.

[8] R. Kannan, Some results on fixed points, Bull. Calcutta Math. Soc. 60 (1968), 71-76. 
[9] H. Kunzi, Nonsymmetric distances and their associated topologies: About the origins of basic ideas in the area of asymmetric topology, in: Handbook of the History of General Topology (eds. C.E. Aull and R. Lowen), vol. 3, Kluwer Acad. Publ. (Dordrecht, 2001), 853-968.

[10] S. Matthews, Partial metric topology, in: Proc. 8th Summer Conference on General Topology and Applications. Ann. New York Acad. Sci. 728 (1994), 183-197.

[11] S. Oltra, O. Valero, Banach's fixed point theorem for partial metric spaces, Rend. Ist. Mat. Univ. Trieste. 36 (2004), 17-26.

[12] S. O'Neill, Partial metrics, valuations and domain theory, in: Proc. 11th Summer Conference on General Topology and Applications. Ann. New York Acad. Sci. 806 (1996), 304-315.

[13] B. Rhoades, A comparison of various definitions of contractive mappings, Trans. Amer. Math. Soc. 226 (1977), 257-290.

[14] S. Romaguera, M. Schellekens, Duality and quasi-normability for complexity spaces, Appl. Gen. Topol. 3 (2002), 91-112.

[15] S. Romaguera, M. Schellekens, Weightable quasi-metric semigroups and semilattices, In: Proc. MFCSIT2000, Electronic Notes in Theoretical Computer Science. 40 (2003), 12 pages.

[16] M. Schellekens, A characterization of partial metrizability: domains are quantifiable, Theoret. Comput. Sci. 305 (2003), 409-432.

[17] M. Schellekens, The correspondence between partial metrics and semivaluations, Theoret. Comput. Sci. 315 (2004), 135-149.

[18] O. Valero, On Banach fixed point theorems for partial metric spaces, Appl. Gen. Topol. 6 (2) (2005), 229-240.

[19] S. Z. Wang, B. Y. Li, Z. M. Gao, K. Iseki, Some fixed point theorems for expansion mappings, Math. Japonica. 29 (1984), 631-636.

[20] X. Wen, X. J. Huang, Common fixed point theorem under contractions in partial metric spaces, J. Comput. Anal. Appl. 13(3) (2011), 583-589.

\footnotetext{
Xianjiu Huang,

Department of Mathematics,

Nanchang University

Nanchang, 330031, Jiangxi, P.R. China

E-mail: xjhuangxwen@163.com
} 
Chuanxi Zhu,

Department of Mathematics,

Nanchang University

Nanchang, 330031, Jiangxi, P.R. China

E-mail: chuanxizhu@126.com

Xi Wen,

Department of Computer Sciences

Nanchang University

Nanchang, 330031, Jiangxi, P.R. China

E-mail: ncuxwen@163.com 